Ferrata Storti Foundation

\title{
Identification of an immunogenic DKK1 long peptide for immunotherapy of human multiple myeloma
}

\author{
Rong Li, ${ }^{1,2,3}$ Chengyun Zheng, ${ }^{4}$ Qiang Wang, ${ }^{1^{\circ}}$ Enguang $\mathrm{Bi}^{1^{\circ}}{ }^{\circ}$ Maojie Yang, ${ }^{1^{\circ}}$ \\ Jian Hou, ${ }^{5}$ Weijun Fu, ${ }^{2}$ Qing $\mathrm{Yi}^{1^{\circ}}$ and Jianfei Qian ${ }^{1^{\circ}}$ \\ ${ }^{1}$ Department of Cancer Biology, Lerner Research Institute, Cleveland Clinic, OH, USA; \\ ${ }^{2}$ The Center of Lymphoma and Multiple Myeloma, ChangZheng Hospital, The Second \\ Military Medical University, Shanghai, P. R. China; ${ }^{3}$ Navy Medical Center of PLA, \\ Shanghai, P. R. China; ${ }^{4}$ Department of Hematology, Second Hospital of Shandong \\ University, Jinan, P. R. China and ${ }^{5}$ Renji Hospital, Shanghai Jiao Tong University School \\ of Medicine, Shanghai, P.R. China
}

Haematologica 2021

Volume 106(3):838-846

${ }^{\circ}$ Current address: Center for Translational Research in Hematologic Malignancies, Houston Methodist Cancer Center/Houston Methodist Research Institute, Houston, TX, USA

\section{ABSTRACT}

$\mathrm{D}$ ickkopf-1 (DKK1), broadly expressed by tumor cells from human multiple myeloma (MM) and other cancers but absent from most normal tissues, may be an ideal target for immunotherapy. Our previous studies have shown that DKK1 (peptide)-specific cytotoxic T lymphocytes can effectively lyse primary MM cells in vitro. To develop DKK1-based vaccines that can be easily and inexpensively made and used by all patients, we identified a DKK1 long peptide (LP), DKK1 $1_{3-76}$ LP, that contains 74 amino acids and epitopes that can potentially bind to all major MHC class I and II molecules. Using HLA-A*0201- and HLA-DR*4-transgenic mouse models, we found that DKK1-specific $\mathrm{CD} 4^{+}$and $\mathrm{CD}^{+} \mathrm{T}$-cell responses, detected by DKK1 short peptide (P20 and P66v)-HLA-A*0201 tetramer staining and cytotoxic assay for $\mathrm{CD}^{+} \mathrm{T}$ cells or by carboxyfluorescein diacetate succinimidyl ester (CSFE) dilution and IFN- $\gamma$ secretion for $\mathrm{CD}^{+} \mathrm{T}$ cells, respectively, can be induced in vivo by immunizing mice with the $\mathrm{DKK} 1_{3-76}$-LP. In addition, $\mathrm{DKK} 1_{3-76}$ LP also induced anti-DKK1 humoral immunity in the transgenic mice and the DKK1 antibodies were functional. Finally, DKK1 $1_{3-76}$-LP stimulated human blood $\mathrm{T}$ cells ex vivo to generate $\mathrm{DKK} 1$-specific $\mathrm{CD}^{+}$and $\mathrm{CD}^{+}$ T-cell responses from 8 out of $10 \mathrm{MM}$ patients with different MHC backgrounds. The generated DKK1-specific $\mathrm{CD}^{+}$cells efficiently lysed autologous MM cells from these patients. Thus, these results confirm the immunogenicity of the $\mathrm{DKK}_{3-76^{-} \mathrm{LP}}$ in eliciting DKK1-specific $\mathrm{CD}^{+}{ }^{+}$and $\mathrm{CD} 8^{+} \mathrm{T}$-cell responses in vitro and in vivo, and suggest that the $\mathrm{DKK}_{3-76}$ - LP can be used for immunotherapy of $\mathrm{MM}$ and other cancers.

Accepted: February 12, 2020.

Pre-published: February 20, 2020.

https://doi.org/10.3324/haematol.2019.236836

(C)2021 Ferrata Storti Foundation

Material published in Haematologica is covered by copyright. All rights are reserved to the Ferrata Storti Foundation. Use of published material is allowed under the following terms and conditions:

https://creativecommons.org/licenses/by-nc/4.0/legalcode. Copies of published material are allowed for personal or internal use. Sharing published material for non-commercial purposes is subject to the following conditions:

https://creativecommons.org//icenses/by-nc/4.0/legalcode, sect. 3. Reproducing and sharing published material for commercial purposes is not allowed without permission in writing from the publisher.

\section{Introduction}

Dickkopf-1 (DKK1) is highly expressed in tumor cells of multiple myeloma (MM) and other cancer types, ${ }^{1,2}$ but is absent from normal tissues and organs, with the exception of the placenta and prostate. ${ }^{3,4}$ Our previous studies have shown that DKK1 (peptide)-specific cytotoxic T lymphocytes (CTL) can effectively lyse primary myeloma cells in vitro, confirming that DKK1 may be a good tumor-associated antigen. We explored the efficacy of a murine DKK1 DNA vaccine in the murine MOPC-21 myeloma model and showed that active vaccination using the DKK1 vaccine was not only able to protect mice from developing myeloma, but was also therapeutic against established myeloma. Mechanistic studies revealed that the DKK1 vaccine elicited strong DKK1- and tumor-specific $\mathrm{CD}^{+}$and $\mathrm{CD}^{+}$immune responses. ${ }^{5}$ Thus, our studies provide a strong rationale for targeting DKK1 for immunotherapy in myeloma patients.

There has been substantial progress in the clinical use of long peptide (LP) therapeutic vaccination in recent years. ${ }^{67}$ Melief et al. reported an LP vaccine encompassing 
a CTL epitope and possessing immunotherapeutic potential. Disis et al. reported that vaccination with a CTL-epitope LP derived from human epidermal growth factor receptor 2 (EGFR2, better known as HER2) generated robust and persistent tumor-specific T-cell immunity in patients with metastatic breast cancer. ${ }^{8}$ Recent clinical studies using a telomerase-derived LP encompassing CTL-epitopes (GV1001) showed an increase in survival of cancer patients when given in combination with radio- and chemotherapy. ${ }^{9}$ The success of LP therapeutic vaccines can be attributed to the fact that it could induce close collaboration between cells of the innate immune system, in particular antigen-presenting dendritic cells (DC) and cells of the adaptive immune system, especially CD4 ${ }^{+}$T-helper (Th) cells and $\mathrm{CD} 8{ }^{+} \mathrm{CTL} .{ }^{10} \mathrm{LP}$ must be taken up and processed by antigen-presenting cells (APC) before they are presented. Professional APC, such as DC, can manage pools of LP and are capable of properly excising multiple HLA class I and II peptide epitopes for presentation at the cell surface. ${ }^{11-13}$ Therefore, injection of LP will ensure the induction of both $\mathrm{CD}^{+}$and $\mathrm{CD}^{+} \mathrm{T}$ cells to available epitopes, each of which can contribute to the anti-tumor response. CD ${ }^{+}$CTL exert key cytotoxicity to tumor cells. $\mathrm{CD}^{+} \mathrm{T}$ cells are necessary elements of cellular immunity for priming tumor-specific CTL and influencing the differentiation and expansion of tumor antigen-specific CTL. Thus, an ideal peptide vaccine for cancer immunotherapy may be optimally composed of a single LP spanning epitopes for both Th cells and CTL.

In this study, we identified a human DKK1-derived LP $\left(\mathrm{DKK} 1_{3-76} \mathrm{LP}\right)$ and explored its potential as a vaccine to induce human DKK1-specific $\mathrm{CD}^{+} \mathrm{Th}$ and $\mathrm{CD}^{+} \mathrm{CTL}$ responses. We found that $\mathrm{DKK} 1_{3-76}$-LP successfully induced Th1-cell responses in individuals expressing several common HLA allelic variants, including HLA-A, HLA-B, HLA-C, $H L A-D R$ alleles, and that an efficient cross-presentation of the DKK $1_{3-76}$-LP also induced DKK1-specific CTL response.

\section{Methods}

\section{Patients and samples}

Peripheral blood samples from patients with $\mathrm{MM}$ and healthy donors were used. This study was approved by the Institutional Review Board of the Cleveland Clinic, and informed consent was obtained in accordance with the Declaration of Helsinki.

\section{Selection of HLA class I- and class II-binding peptides}

To predict possible promiscuous HLA class I and II-binding peptides on human DKK1, the amino acid sequence of the human DKK1 protein was analyzed by Immune Epitope Database (IEBD) recommended methods. ${ }^{14}$ The program identified a 74 amino acid LP, DKK1 $1_{3-76}$, that contains multiple peptide motifs (Figure 1) with high affinity for common and major MHC class I and class II molecules, representing $95 \%$ of humans. ${ }^{14}$

All peptides, including long and short MHC class I and class II binding peptides, were synthesized by Biosynthesis (Lewisville, TX, USA). The purity of synthetic peptides, confirmed by reversed-phase high-performance liquid chromatography and mass spectrometry, was over $98 \%$. Synthetic peptides were dissolved in dimethyl sulfoxide (DMSO; Sigma, St Louis, MO, USA), and stored at $-20^{\circ} \mathrm{C}$ until use.

\section{Generation of dendritic cells}

Monocyte-derived mature DC were generated from human peripheral blood mononuclear cells (PBMC) ${ }^{11,15}$ The quality of
DC was judged based on their expression of CD11c, CD40, CD80, CD86, and MHC class II molecules. ${ }^{16}$ Detailed information is provided in the Online Supplementary Appendix.

\section{Determination of in vivo immunogenicity of DKK1 peptides}

HLA-A*0201-transgenic (Tg[HLA-A2.1]) ${ }^{17}$ and HLA-DR*4transgenic mice were purchased from the Jackson Laboratory (Bar Harbor, ME, USA). ${ }^{18}$ Mice were maintained at the animal facility and studies were approved by the Institutional Animal Care and Use Committee of the Cleveland Clinic.

For immunization, peptides were diluted in phosphate buffered saline at room temperature, mixed, and emulsified with an equal volume of incomplete Freund's adjuvant (Sigma). Groups of three mice were immunized subcutaneously at the tail base with $100 \mathrm{~L}$ of emulsion containing $100 \mathrm{~g}$ of peptides. All of the mice were immunized at least three times. Two weeks after the immunization, mice were killed and splenocytes were isolated for in vitro studies. The same experiments were repeated three times.

\section{Generation of DKK1-specific CD4 ${ }^{+}$and $\mathrm{CD} 8^{+} \mathrm{T}$-cell responses}

DKK1-specific $T$ cells were generated from PBMC of HLA$\mathrm{A}^{*} 0201^{+}$and HLA-DR ${ }^{*}{ }^{+}$blood donors and patients with MM by repeated stimulations of autologous T cells with DKK1 peptide-loaded mature DC. Further details are available in the Online Supplementary Appendix.

\section{Cytotoxicity assay}

The standard 7-AAD/CFSE Cell-Mediated Cytotoxicity Assay Kit was used to measure the cytolytic activity of $\mathrm{T}$ cells on target cells. Further details are available in the Online Supplementary Appendix.

\section{Assessment of DKK1-specific T-cell responses}

The frequency of peptide-specific, IFN- $\gamma$-secreting $\mathrm{CD}^{+} \mathrm{T}$ cells was analyzed using $3 \times 10^{4}$ bulk $\mathrm{CD}^{+} \mathrm{T}$ cells stimulated with equal numbers of peptide-pulsed autologous $\mathrm{PBMC}$ or, alternatively, $5 \times 10^{4}$ bulk $\mathrm{CD} 4^{+} \mathrm{T}$ cells stimulated with $1 \times 10^{4}$ peptide-pulsed DC expressing HLA-DR or -DP molecules. Further details are available in the Online Supplementary Appendix.

\section{Statistical analyses}

Statistical analysis was performed with Student $t$-test. $P<0.05$ was considered statistically significant. Results are presented as mean \pm standard deviation unless otherwise indicated.

\section{Results}

Identification of long peptide containing multiple T-cell epitopes on Dickkopf-1 protein

To identify LP comprising the most potential MHC class I and II binding epitopes on human DKK1 protein, we examined the amino acid sequence of DKK1 using the following websites: http://www.bimas.cit.nih.gov/molbio/hla bindl, http://www.imtech.res.in/raghavalpropred/, www. immuneepitope.org to predict the epitopes. We focused on regions with multiple MHC class I and class II epitope binding prospects. As a result, we identified an LP, $\mathrm{DKK}_{3-76}$, that contains 74 amino acids and multiple epitopes that can potentially bind with all major MHC class I (e.g., HLA-A, B, or C) and class II molecules (e.g., HLA- 
Table 1. Potential Dickkopf-1 peptides for different MHC molecules.

\begin{tabular}{|c|c|c|c|c|}
\hline $\begin{array}{l}\text { MHC class I } \\
\text { HLA }\end{array}$ & Name & Sequence & Position & Predictive binding ${ }^{\text {a.b.c. }}$ \\
\hline \multirow[t]{10}{*}{ A0201 } & P3 & ALGAAGATRV & 3 & 70 \\
\hline & Рy3 & YLGAAGATRV & 3 & 320 \\
\hline & Pyllv & RVFVAMVAA & 11 & 238 \\
\hline & $\mathrm{P} 20$ & ALGGHPLLGV & 20 & 160 \\
\hline & Py20 & YLGGHPLLGV & 20 & 736 \\
\hline & Py25 & YLLGVSATL & 25 & 364 \\
\hline & Py32v & YLNSVLNSNV & 32 & 320 \\
\hline & P 36 & VLNSNAIKNL & 36 & 84 \\
\hline & P36v & VLNSNAIKNV & 36 & 226 \\
\hline & P66v & ILYPGGNKV & 66 & 378 \\
\hline $\mathrm{Al}$ & Py35v & YVLNSNAIV & 35 & 42.5 \\
\hline \multirow[t]{2}{*}{ A0205 } & Pyllv & YVFVAMVAV & 11 & 72 \\
\hline & $\mathrm{P} 13$ & FVAMVAAAL & 13 & 42 \\
\hline A24 & $\mathrm{P} 12$ & VFVAmVAAAL & 12 & 42 \\
\hline B62 & $\mathrm{P} 66$ & ILYPGGNKY & 66 & 124.8 \\
\hline \multirow[t]{2}{*}{ B7 } & P24 & HPLLgVSATL & 24 & 80 \\
\hline & P59 & AVSAAPGIL & 59 & 60 \\
\hline B5101 & P68 & YPGGnKYQTI & 68 & 692 \\
\hline B5102 & P68 & YPGGnKYQTI & 68 & 1064.8 \\
\hline \multirow[t]{2}{*}{ B5103 } & P5 & GAAGaTRVFV & 5 & 121 \\
\hline & P51 & GAAGhPGSAV & 51 & 110 \\
\hline B5201 & P4 & LGAAgATRVF & 4 & 74.3 \\
\hline \multirow[t]{2}{*}{ CW0401 } & $\mathrm{P} 12$ & VFVAmVAAAL & 12 & 240 \\
\hline & P24 & HPLLgVSATL & 24 & 96 \\
\hline \multicolumn{5}{|l|}{ MHC class II } \\
\hline $\mathrm{DRB1}$ *0101 & P10 & TRVFVAMVAAALGGH & 10 & 33 \\
\hline $\mathrm{DRB1} 1 * 0301$ & P26 & LLGVSATLNSVLNSN & 26 & 25 \\
\hline $\mathrm{DRB1}$ *0401 & P30 & SATLNSVLNSNAIKN & 30 & 26 \\
\hline DRB1*0701 & P26 & LLGVSATLNSVLNSN & 26 & 30 \\
\hline DRB1*1501 & $\mathrm{P} 7$ & AGATRVFVAMVAAAL & 7 & 24 \\
\hline
\end{tabular}

ahttp://www.bimas.cit.nih.gov/molbio/hla_bind. ${ }^{\mathrm{b}}$ http://www.imtech.res.in/raghava/propred/. chttp://www.immuneepitope.org.

DR1, -DR4, or -DR7) (Table 1 and Figure 1). DKK1 $1_{3-76}$-LP contains our previously identified HLA-A*0201-restricted T-cell epitopes DKK1-P20 and DKK1-P66v. ${ }^{19}$

\section{In vivo immunogenicity of the Dickkopf-1 ${ }_{3-76}$-long peptide in activating Dickkopf-1-specific CD $8^{+}$ cytotoxic T lymphocytes}

To assess the immunity of the $\mathrm{DKK} 1_{376} \mathrm{LP}$ in inducing $\mathrm{CD}^{+}$CTL response in vivo, we used HLA-A*0201-transgenic mice and immunized them four times with either DKK1 $1_{3-76}$-LP or DKK1-P20 short peptide. The capacity of DKK1 $1_{3-76}$-LP to prime DKK1-specific CTL response was examined using HLA-A*0201-P20- (Figure 2A) or HLA$\mathrm{A}^{*} 0201-\mathrm{P} 66 \mathrm{v}$ tetramer staining (Figure $2 \mathrm{~B}$ ). The results clearly showed that mice immunized with DKK1-P20 short peptide (Figure $2 \mathrm{~A}$ and $\mathrm{B}$, top panels) had increased percentages of DKK1-P20 $(P<0.01$, compared with control), but not DKK1-P66v, tetramer ${ }^{+} \mathrm{CD}^{+} \mathrm{T}$ cells in the spleen after each round of immunization, whereas mice immunized with $\mathrm{DKK}_{3-76}$-LP (Figure $2 \mathrm{~A}$ and $\mathrm{B}$, bottom panels) generated $\mathrm{CD}^{+} \mathrm{T}$-cell response against both
DKK1-P20 and DKK1-P66v $(P<0.01$, compared with control). Moreover, $\mathrm{CD}^{+} \mathrm{T}$ cells isolated from mice immunized with $\mathrm{DKK} 1_{3-76} \mathrm{LP}$ were not only able to kill syngeneic DC pulsed with $\mathrm{DKK} 1_{3-76}-\mathrm{LP}$, but also DC pulsed with DKK1-P20 short peptide $(P<0.01$, compared with controls) (Figure 2C). Furthermore, these $\mathrm{CD}^{+} \mathrm{T}$ cells also killed HLA-A*0201+ U266, but not HLA-A*0201 ARP-1 myeloma cells or K562 cells (to exclude natural killer [NK]-cell activity) $(P<0.01$, compared with controls) (Figure $2 \mathrm{D})$. Hence, these results demonstrate that DC, after uptake of DKK $1_{3-76}$-LP, efficiently cross-present $\mathrm{T}$-cell epitopes on the DKK $1_{3-76}$-LP and activate CTL specific for various Tcell epitopes, including DKK1-P20- and DKK1-P66v, in vivo.

\section{In vivo immunogenicity of the Dickkopf-1 ${ }_{3 \cdot 76}$-long} peptide in activating Dickkopf-1-specific CD4 ${ }^{+}$-helper cells and antibody production

Next, we assessed whether DKK1 $1_{3-76}$-LP could also elicit DKK1-specific CD4+ Th cell response. HLA-DR*4-transgenic mice were available commercially and immunized 

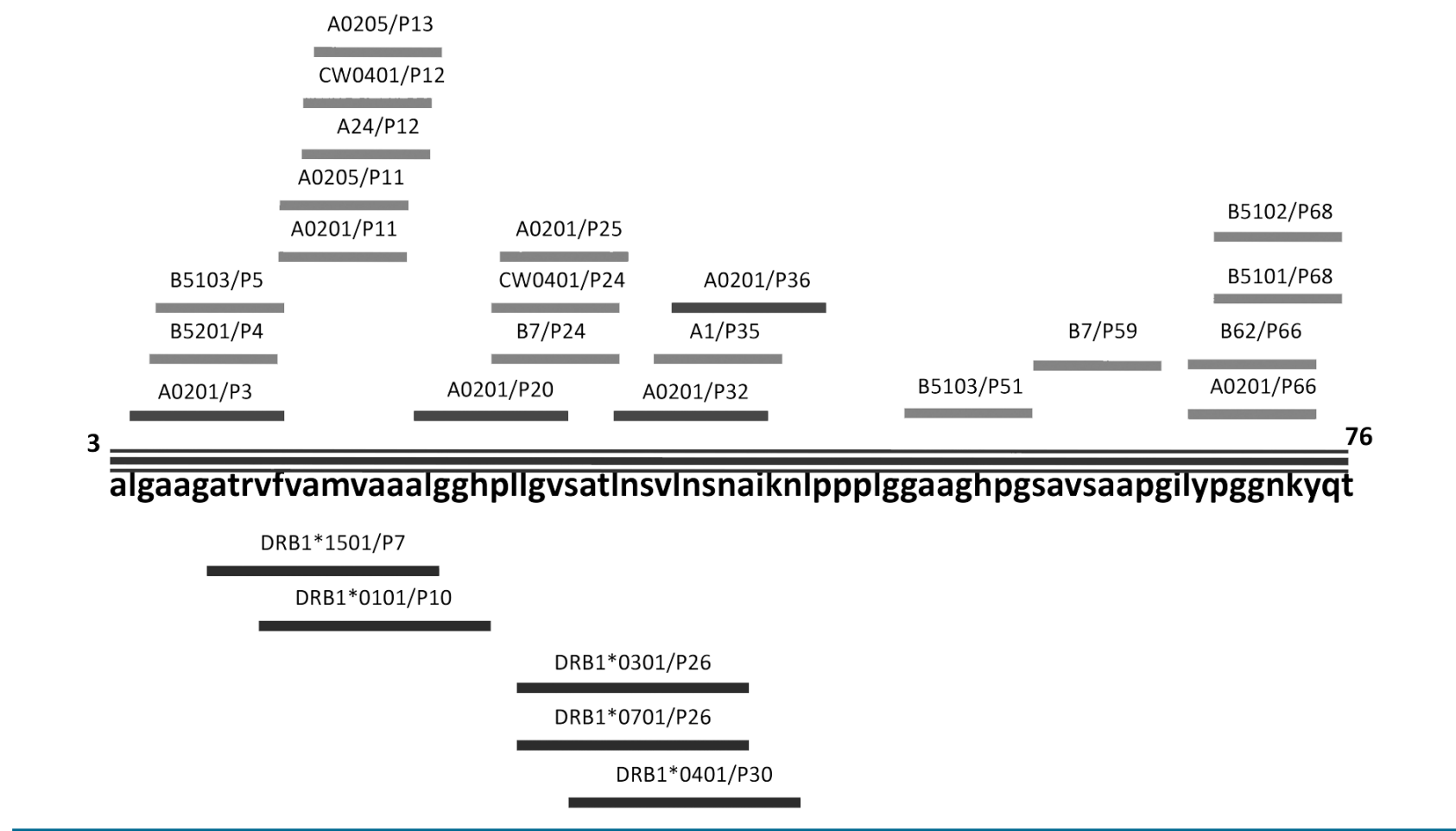

Figure 1. Dickkopf-1 (DKK1) $)_{30}$-long peptide (LP). Schematic presentation of DKK1 $1_{37}$-LP and epitopes for MHC class I and class II molecules.

four times with DKK $1_{3-76}$-LP or a HLA-DR*4-restricted and -binding DKK1-P30 short peptide (Table 1). CD4+ T-cell response was detected by CFSE dilution and IFN- $\gamma$ secretion. The results clearly showed that mice immunized with either DKK1 $1_{3-76}$ LP or DKK1 P30 short peptide had significantly higher percentages of proliferating $\mathrm{CD}^{+} \mathrm{T}$ cells in the spleen after ex vivo re-stimulation with DC pulsed, but not unpulsed, with DKK1 $1_{3-76}$ LP or DKK1-P30 short peptide $(P<0.01$, compared with non-immunized mice) (Figure $3 \mathrm{~A}$ ). Moreover, splenocytes isolated from DKK1 $1_{3-76}$ LP - or DKK1 P30-immunized mice contained significantly more $\mathrm{CD} 4^{+}$IFN- $\gamma$-expressing cells after ex vivo re-stimulation with $\mathrm{DKK} 1_{3-76}$-LP - or DKK1 P30-, respectively, pulsed, but not unpulsed, DC than non-immunized mice $(P<0.01$, compared with those from non-immunized mice) (Figure $3 \mathrm{~B}$ ). Interestingly, the percentages of Foxp $3^{+} \mathrm{CD} 4^{+} \mathrm{T}$ cells were similarly low in mice with or without peptide immunization (Figure $3 \mathrm{~B}$ ), indicating that $\mathrm{DKK} 1_{3-76}$-LP vaccination induced DKK1-specific CD4 $4^{+} \mathrm{T}$ cell responses without promoting regulatory $\mathrm{T}$-cell (Treg) formation in vivo. Taken together, these results demonstrate that the $\mathrm{DKK} 1_{3-76} \mathrm{LP}$ is immunogenic in vivo to induce DKK1-specific CD $4^{+}$and $\mathrm{CD}^{+} \mathrm{T}$-cell responses.

We also investigated whether the $\mathrm{DKK}_{3-76} \mathrm{LP}$ could induce a DKK1-specific humoral immune response and examined whether there were DKK1-specific antibodies in the sera of $\mathrm{DKK}_{3-76}$-LP-immunized HLA-A*0201- or HLA-DR*4-transgenic mice. ELISA results showed that high titers of DKK1-specific IgG antibodies were detected in HLA-A*0201-transgenic mice immunized with DKK1 $1_{3-76}$-LP, but not with DKK1 P20 short peptide, and the titers of the antibodies increased after each cycle of immunization $(P<0.01$, compared with DMSO control) (Figure 4A). Similarly, DKK1-specific antibodies were also detected in the sera of HLA-DR*4-transgenic mice immunized with DKK1 $1_{3-76}$-LP but not the DKK1-P30 short peptide $(P<0.01$, compared with DMSO control) (Figure $4 \mathrm{~B})$.

Next, we determined whether the detected DKK1-specific antibodies in $\mathrm{DKK}_{3-76}$-LP-immunized mice were biologically functional. As DKK1 was shown to inhibit human osteoblast differentiation, ${ }^{20}$ we used an in vitro osteoblast culture system to determine the function of the immunized sera on osteoblast formation in the presence of recombinant human DKK1. The results showed that commercially obtained DKK1-specific antibodies and sera from DKK $1_{3-76}$-LP-immunized transgenic mice abolished DKK1-induced inhibition of human osteoblast differentiation (Figure 4C). These results indicate that the DKK $1_{3-76}$-LP encompasses naturally processed B-cell epitopes, and antibodies generated by $\mathrm{DKK} 1_{3-76} \mathrm{LP}$ immunization are able to neutralize DKK1.

Immunogenicity of the Dickkopf-1 $\mathbf{1}_{3-76}$-long peptide in priming human Dickkopf-1-specific T cells ex vivo

Next, we investigated whether DKK $1_{3-76}$-LP was able to induce human DKK1-specific T-cell responses ex vivo. Freshly prepared PBMC from healthy donors or myeloma patients were stimulated with $\mathrm{DKK} 1_{3-76}$-LP every week. The presence and frequency of DKK1-specific T cells were detected by flow cytometry. Figure 5A shows an increased frequency of DKK1-specific, IFN- $\gamma$-secreting $\mathrm{CD}^{+}$and $\mathrm{CD}^{+}$cells in cultured $\mathrm{T}$ cells during in vitro (re)-stimulation with the LP. Figure 5B shows the percentages of HLAA*0201-DKK1-P20 tetramer $^{+} \mathrm{CD}^{+}{ }^{+} \mathrm{T}$ cells in cultures after repeated stimulations with $\mathrm{DKK} 1_{3-76}$-LP. By using monoclonal antibodies (mAb) specific to HLA-DR or -DQ or HLA-ABC added to T-cell cultures before assay, we showed that $\mathrm{MHC}$ class II-restricted $\mathrm{CD}^{+} \mathrm{T}$ cells were 
A

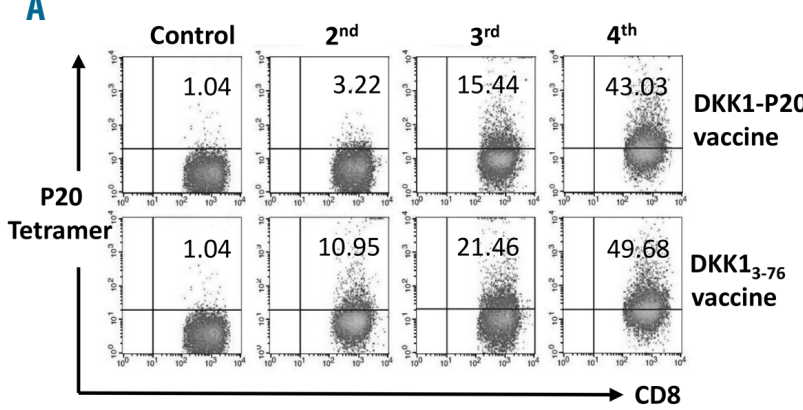

B

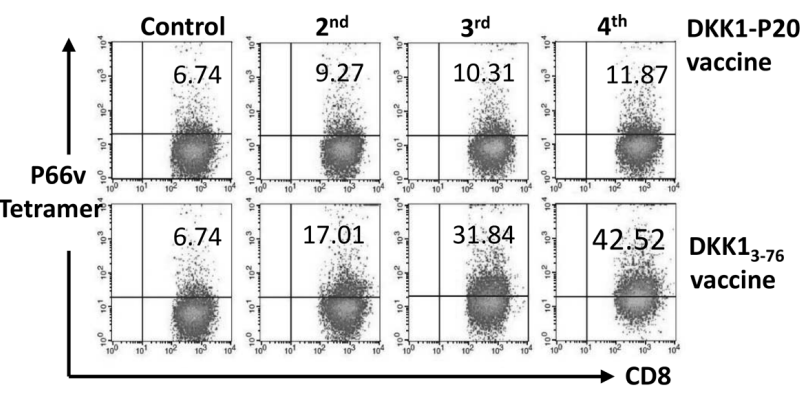

D

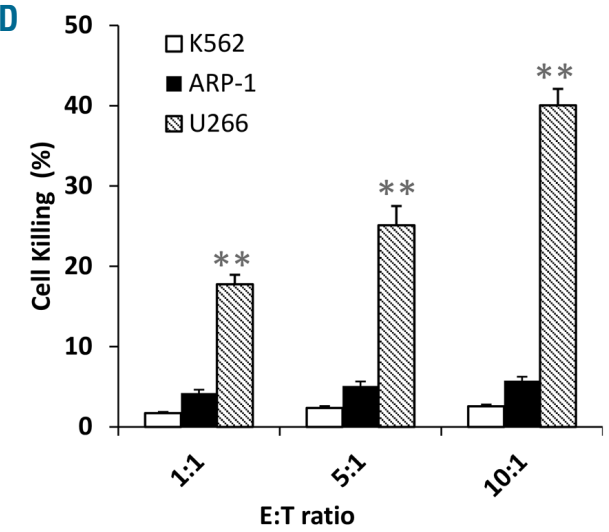

Figure 2. Cross-presentation of Dickkopf-1 (DKK1) $)_{3 \pi}$-long peptide (LP) efficiently primes DKK1-specific CD8 ${ }^{+} \mathrm{T}_{\text {cells }}$ in vivo. Shown are CD8 ${ }^{+} \mathrm{T}_{-\mathrm{cell}}$ responses induced in HLA-A*0201-transgenic mice after immunization with DKK1 $1_{3 n}$-LP or HLA-A*0201-restricted P20 short peptide. (A) DKK1-P20-specific tetramer staining showing the frequency of DKK1 P20-specific CD8 ${ }^{+} T$ cells in the spleen of a HLA-A*0201-transgenic mouse. Representative results from one of three mice are shown. (B) DKK1-P66v-specific tetramer staining showing the frequency of DKK1 P66v-specific CD8 ${ }^{+} T$ cells in the spleen of an HLA-A*0201-transgenic mouse. These results indicate that DKK1 ${ }_{2 \pi}$-LP was cross-presented and efficiently primed DKK1-P66v-specific CD8 ${ }^{+} \mathrm{T}$ cells in HLA-A*0201-transgenic mice. Representative results from one of three mice are shown. (C) Cytolytic activity of CD8 ${ }^{+} \mathrm{T}$ cells isolated from mice immunized with DKK $1_{3 \pi} \mathrm{LP}$ against unpulsed dendritic cells (DC) or DC pulsed with DKK1 $1_{35} \mathrm{LP}$ or P20 short peptide. Representative results of three experiments are shown. (D) Cytolytic activity of CD8 ${ }^{+} \mathrm{T}$ cells isolated from mice immunized with DKK $1_{376}$ LP against U266 and ARP-1 human myeloma cell lines, and K562. Representative results of three experiments are shown. $* P<0.05 ; * \star P<0.01$.

the main IFN- $\gamma$-secreting cells, because anti-HLA-DR mAb significantly reduced the percentage of IFN- $\gamma$-secreting $\mathrm{CD}^{+}$cells (Figure $5 \mathrm{C}$ ). Moreover, HLA-A2 antibody significantly reduced the percentages of IFN- $\gamma$-secreting $\mathrm{CD}^{+} \mathrm{T}$ cells in cultures after repeated stimulations with DKK $1_{3-76}$-LP. The results showed that DKK1 $1_{3-76}$-LP-specific $\mathrm{CD}^{+}$T-cell responses were HLA*0201-restricted (Figure 5D). Taken together, the results demonstrate that human DKK1-specific CTL and Th1 cells can be induced by DKK1 $1_{3-76}$-LP ex vivo.

Finally, we determined whether the $\mathrm{DKK} 1_{3-76}$-LP could induce DKK1-specific HLA-A*0201-restricted and HLA$\mathrm{DR}^{*} 4$-restricted $\mathrm{T}$-cell responses from $\mathrm{MM}$ patients. After 3-4 weeks of stimulating patient-derived PBMC with $\mathrm{DKK}_{3-76}$-LP in vitro, the frequency of DKK1-specifc $\mathrm{CD}^{+}$ CTL and $\mathrm{CD}^{+}$Th1 cells was detected by intracellular IFN- $\gamma$ staining. Figure $6 \mathrm{~A}$ is a representative flow cytometry analysis showing the percentages of IFN- $\gamma$-secreting $\mathrm{CD}^{+}$and $\mathrm{CD}^{+} \mathrm{T}$ cells after re-stimulation with $\mathrm{DKK} 1_{3-76^{-}}$ LP from a MM patient (MM1). Low percentages of IFN- $\gamma$ secreting $\mathrm{CD}^{+}$and $\mathrm{CD} 4^{+} \mathrm{T}$ cells were observed in T-cell cultures re-stimulated with unpulsed DC or DC pulsed with DKK1-P20 short peptide. Figure $6 \mathrm{~B}$ shows the percentages of IFN- $\gamma$-secreting $\mathrm{CD}^{+}{ }^{+}$and $\mathrm{CD}^{+} \mathrm{T}$ cells from a total of ten patients with MM (with different MHC backgrounds) after a 4-week in vitro stimulation of blood T cells with DKK1 $1_{3-76}$-LP-pulsed autologous DC. Furthermore, we generated $\mathrm{CD}^{+} \mathrm{T}$-cell lines from HLA-A*0201 patients by in vitro repeated stimulations with $\mathrm{DKK} 1_{3-76}$-LP and these DKK1-specific T cells killed autologous patient (MM1) myeloma cells, primary myeloma cells from another HLA-A*0201+ patient (MM2), and HLA-A*0201 ${ }^{+}$ myeloma cell line U266. No killing was observed on HLA$\mathrm{A}^{*} 0201^{-}$primary myeloma cells (MM3) or myeloma cell line ARP-1, normal B cells, or K562 cells (to exclude NKcell activity) (Figure 6C).

\section{Discussion}

In this study, we identified a 74 aa $\mathrm{DKK} 1_{3-76}$-LP thast contains multiple epitopes for $\mathrm{CD}^{+}$and $\mathrm{CD}^{+} \mathrm{T}$ cells and explored the potential of using this LP for immunotherapy of human MM. We showed that DKK1-specific CTL, detected by DKK1 short peptide (P20 and P66v)-HLAA*0201 tetramer staining, and DKK1-specific Th cells, detected by IFN- $\gamma$ secretion and CSFE-dilution assay, can be induced by (cross)-presentation of $\mathrm{DKK} 1_{3-76}$-LP in vitro and in vivo. We also verified the presence of DKK1-specific Th1 responses in MM patients.

Recent studies evaluating the CTL repertoire of HPV-16 e6 and e7 oncogenic protein showed complete and lasting regression of end-stage cervical cancer patients after melanoma antigen A3 (MAGE-A3) vaccination with peptide $^{21,22,29-31}$ After immunization, a new wave of antigenspecific CTL clones arose in the peripheral blood, provid- 
A

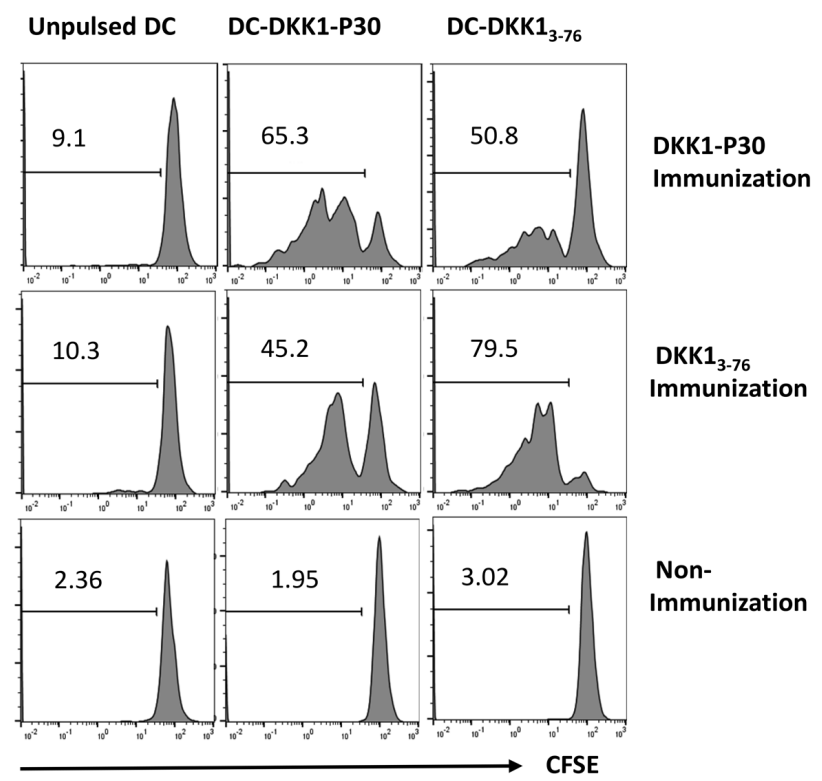

B

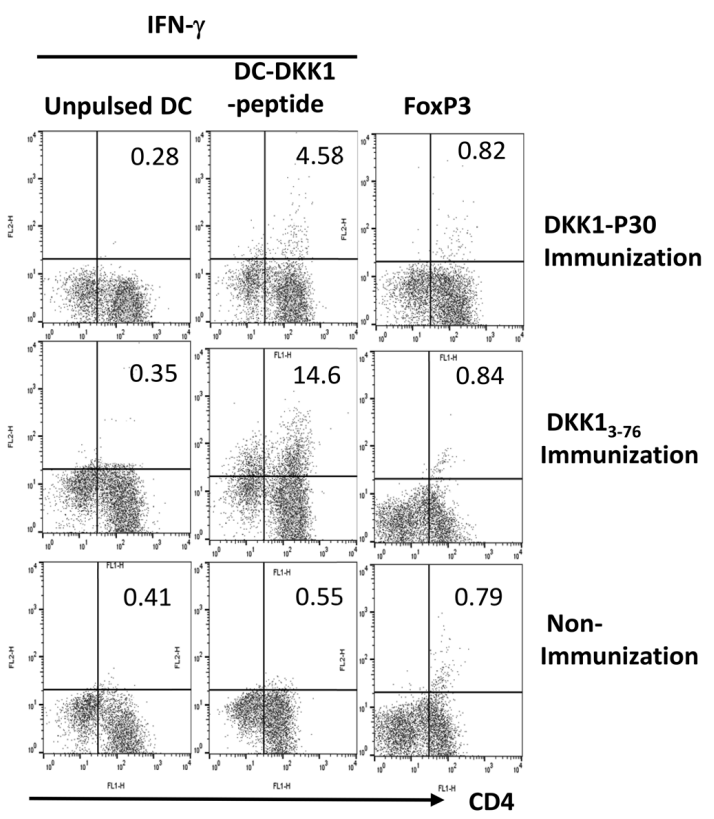

Figure 3. Dickkopf-1 (DKK1) $)_{2 n}$-long peptide (LP) efficiently induces DKK1-specific CD4 ${ }^{+}$T-cell response in vivo. Shown are CD4 ${ }^{+} \mathrm{T}_{-}$-cell responses induced in HLA$\mathrm{DR} * 4$-transgenic mice after immunization with DKK1 $1_{3 \pi}$-LP or HLA-DR-restricted P30 short peptide. (A) Carboxyfluorescein succinimidyl ester (CFSE) dilution assay showing the percentages of proliferating $\mathrm{CD}^{+}{ }^{+} \mathrm{T}$ cells from the spleen of mice after ex vivo re-stimulation with dendritic cells (DC) pulsed, but not unpulsed, with DKK $1_{3 \pi}$ LP or DKK1-P30 short peptide. Representative results from one of three independent experiments are shown. (B) Flow cytometry analysis showing the percentages of IFN- $\gamma$-expressing or FoxP3 ${ }^{+} \mathrm{CD}^{+}{ }^{+}$cells from the spleen of mice after ex vivo re-stimulation with DC pulsed, but not unpulsed, with DKK $1_{3 \pi \mathrm{L}} \mathrm{LP}$ or DKK1P30 short peptide. Representative results from one of four experiments are shown.

A

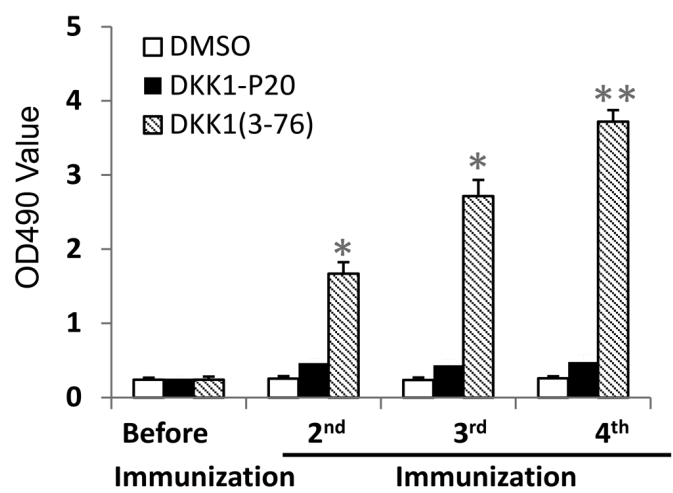

C

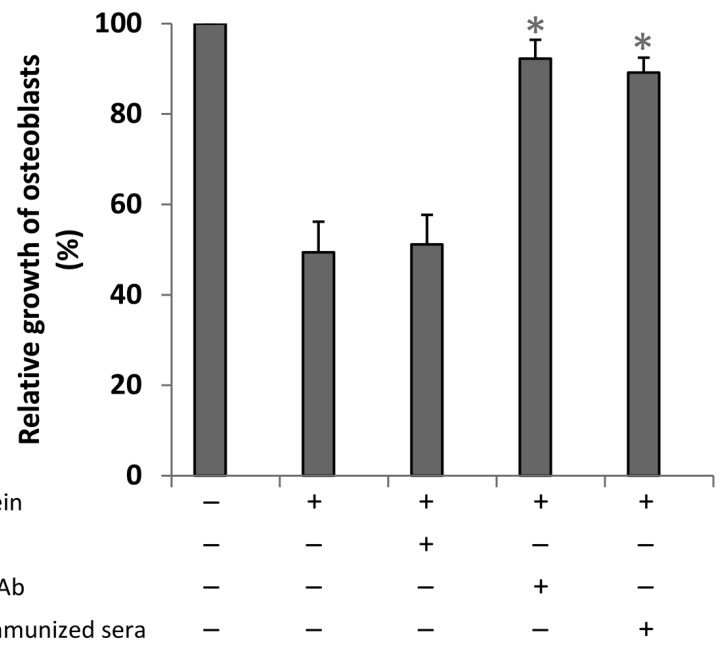

DKK1 $1_{3-76}$ immunized sera
B

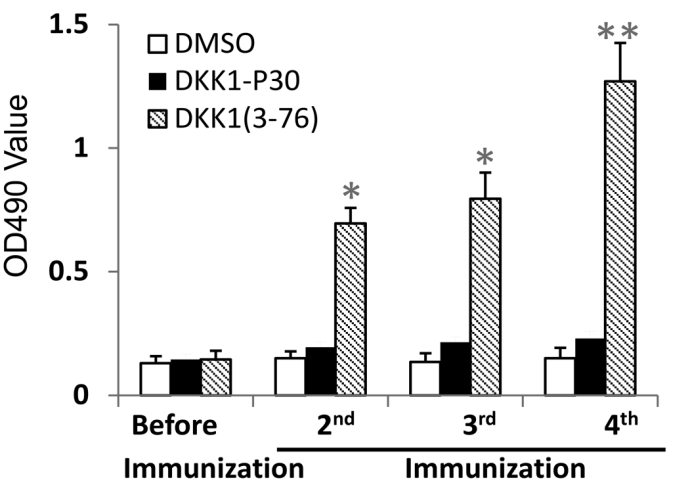

Figure 4. Dickkopf-1 (DKK1) $)_{30}$-long peptide (LP) induces DKK1-specific humoral immune response in vivo. Serum titers of DKK1-specific antibodies in mice after immunization with DKK1 $1_{n-L P}$ or short peptide are shown. (A) ELISA showing the titers of DKK1-specific IgG antibodies in HLA-A*0201-transgenic mice immunized with DKK1 $1_{376}$ LP or P20. (B) ELISA showing the titers of DKK1-specific IgG antibodies in HLA-DR*4transgenic mice immunized with DKK1 LP or P30. (C) Effect of DKK1 on differentiation of human osteoblasts in culture with the addition of control mouse IgG, anti-DKK1 antibody (R\&D Systems) or serum from DKK1. ${ }_{\pi=} \mathrm{LP}$ vaccinated (after the 4th vaccination) HLA-A*0201-transgenic mice. Results of three experiments are shown. DMSO: dimethyl sulfoxide. $\star P<0.05 ; * * P<0.01$. 
A

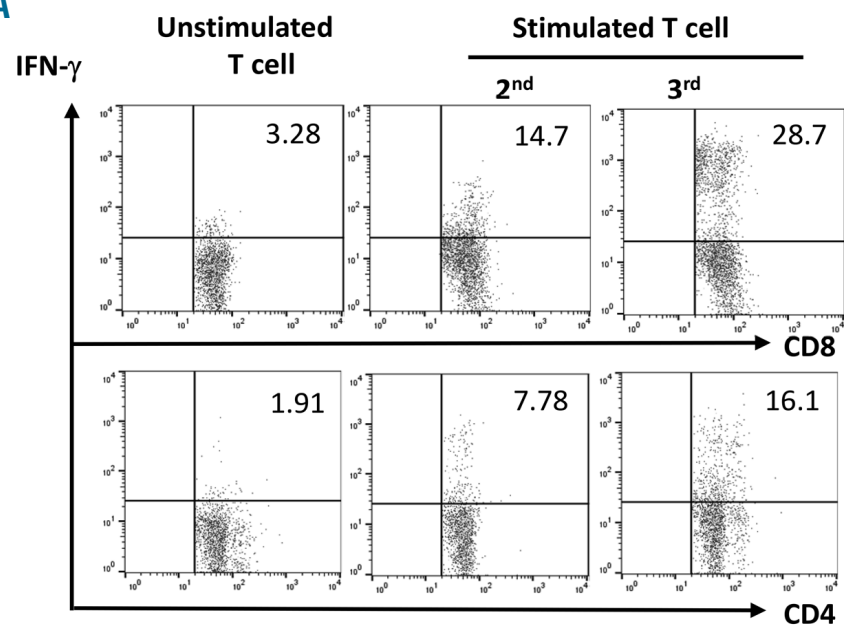

C

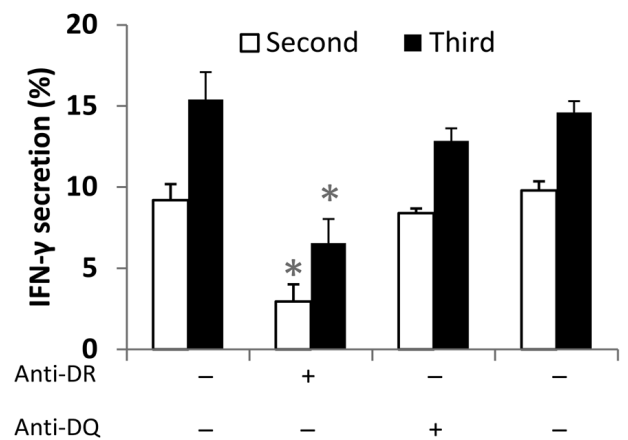

B

Isotype P20 Tetramer

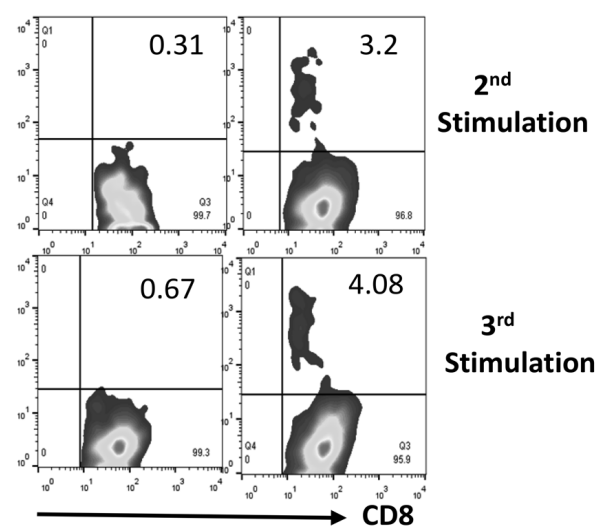

D

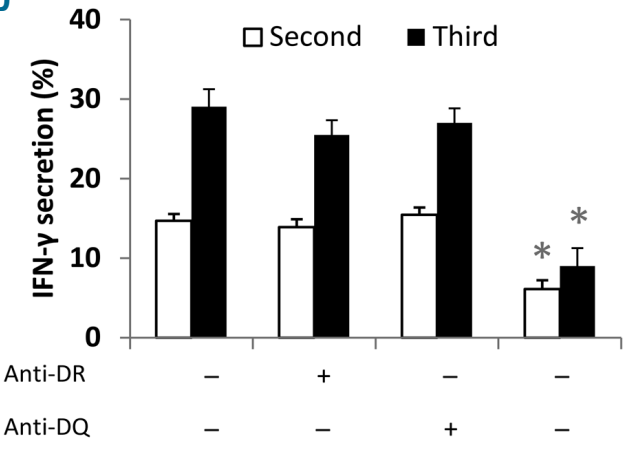

Figure 5. Dickkopf-1 (DKK1) ${ }_{3 \pi}$-long peptide (LP) induces DKK1-specific human T cells in vitro. Fresh peripheral blood mononuclear cells (PBMC) derived from healthy donors (HLA-A0201 ${ }^{+}$or HLA-DR* $4^{+}$) were stimulated with DKK1 $1_{3 \pi}$-LP plus IL-2 and IL-7 weekly in vitro. (A) Frequency of DKK1-specific CD4 ${ }^{+}$and CD8 ${ }^{+}$ IFN- $\gamma$-secreting cells detected by intracellular staining assay. (B) Percentages of HLA-A*0201-DKK1-P20 tetramer ${ }^{+} \mathrm{CD} 8^{+} \mathrm{T}$ cells in culture after second or third in vitro stimulation with DKK $1_{3 \pi}$ LP. (C) MHC class II-restriction of DKK $1_{37}$-LP-specific CD4 $4^{+}$T-cell response. PBMC stimulated with DKK1 $1_{37}$-LP for 1 week were re-stimulated with $\mathrm{DKK} 1_{3 n}$-LP in the presence of different monoclonal antibodies (mAb) specific for HLA-DR, -DQ or HLA-ABC. The results showed that HLA-DR antibody significantly

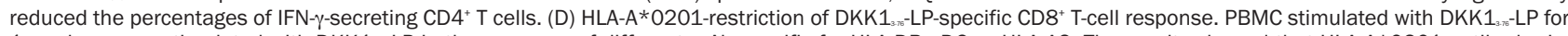
1 week were re-stimulated with DKK1 $1_{3 \pi}$-LP in the presence of different mAb specific for HLA-DR, -DQ or HLA-A2. The results showed that HLA-A*0201 antibody significantly reduced the percentages of IFN- $\gamma$-secreting $\mathrm{CD}^{+} \mathrm{T}$ cells. Representative results of three experiments are shown. ${ }^{*} P<0.05$; ${ }^{*} P<0.01$.

ing solid evidence that the phenomenon of epitope spreading is critical to the development of effective anticancer immunity elicited by peptide vaccination. These results further implicate functional interactions between vaccine-induced CTL and malignant cells that facilitate the induction of large numbers of tumor-specific CTL, the cytolytic effector immune cells that subsequently destroy tumor cells.

Disis et al. reported that vaccination with a herceptin-2 (HER-2/neu)-derived LP encompassing an HLA-A*0201restricted CTL epitope elicited embedded CTL-epitope specific $\mathrm{CD}^{+} \mathrm{T}$ cells in cancer patients. ${ }^{8}$ They showed that tumor-specific CTL can be elicited in vivo via crosspresentation of HER-2/neu-derived LP. Such T-cell responses are considered to be crucial for tumor eradication and for generating long-term memory. ${ }^{23}$ With this premise in mind, we identified an immunogenic DKK1 ${ }_{3-76^{-}}$ LP that encompasses both Th epitopes and CTL-epitopes and demonstrated that cross-presentation of $\mathrm{DKK} 1_{3-76}$-LP induced priming and expansion of DKK1-specific CTL in vitro and in vivo. Vaccination with $\mathrm{DKK} 1_{3-76} \mathrm{LP}$ can potentially elicit combined Th and CTL responses. A recent clinical trial showed that targeting Th cells with DC pulsed with both HLA class I and II-restricted epitopes effectively enhanced vaccine-specific immune responses and improved clinical outcome. ${ }^{24} \mathrm{DKK}_{3-76}$-LP bolstered the induction of DKK1-P20-specific CTL derived from both healthy donors and $\mathrm{MM}$ patients in vitro. Thus, DKK $1_{3-76}$-LP administered in combination with DKK1-P20 immunotherapy may be able to augment the elicitation of antigen-specific CTL.

Based on the HLA-subtypes capable of antigen presentation from studies using healthy donors, DKK1-P20 and $\mathrm{DKK} 1_{3-76}$-LP are predicted to be useful in approximately $80 \%$ of the total population. We showed that $\mathrm{DKK} 1_{3-76}$ - LP induced HLA-DP5-, HLA-DR8-, or HLA-DR15-restricted Th cells in healthy donors and also induced HLA-DR- or HLADQ-restricted Th cells in MM patients. However, these MM patients were negative for HLA-DP5, -DR8, or -DR15 alleles. We also showed that DKK1 $1_{3-76}$-LP induced HLA-DR15- or HLA-DQ-restricted Th cells in healthy donors. These results suggest that DKK1 $1_{3-76}$ LP may encompass Th cell epitopes not previously identified in experiments involving cells derived from healthy donors and $\mathrm{DKK} 1_{3-76} \mathrm{LP}$ may be broadly useful in the majority of MM patients.

Weide et al. reported that the presence of circulating Th cells responding to melanoma antigens Melan-A or NY-ESO-1 has a strong independent prognostic impact on survival among chemotherapy-treated advanced melanoma patients. ${ }^{25}$ Another study has shown a possible 


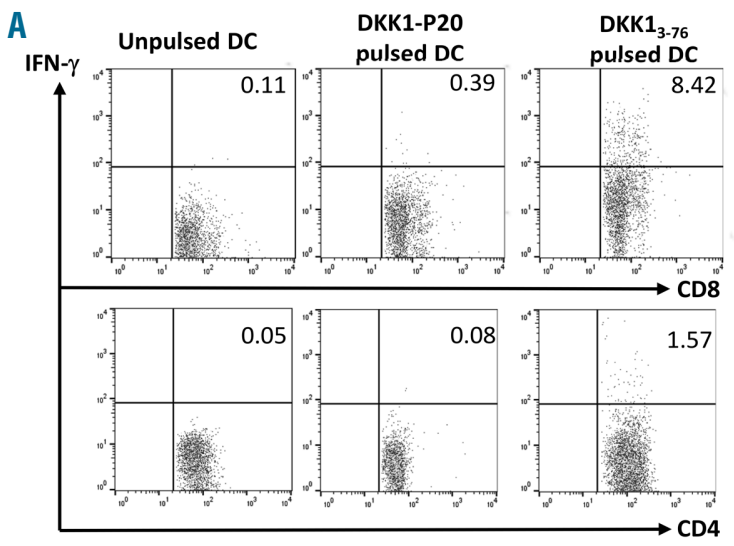

C

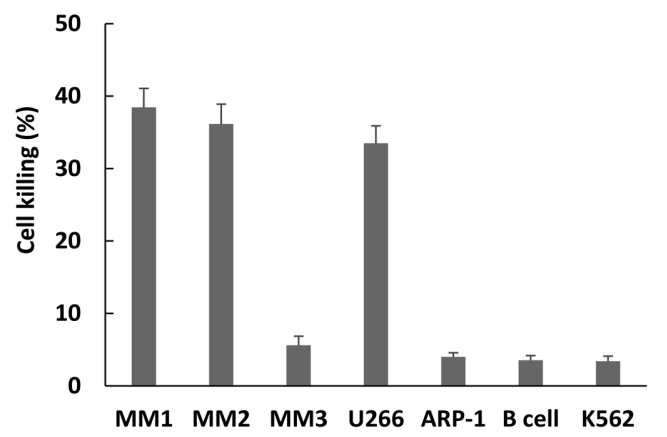

B

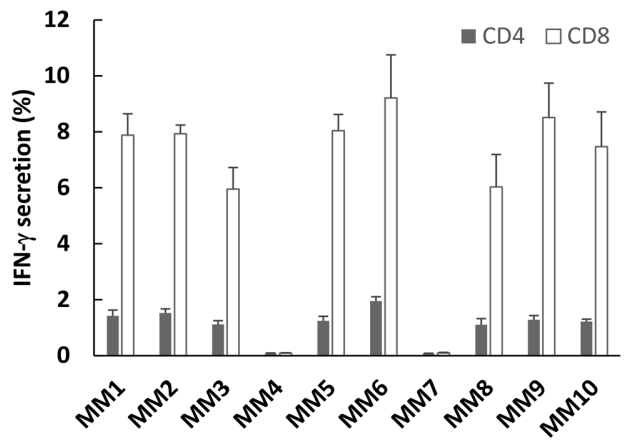

Figure 6. Dickkopf-1 (DKK1) $)_{37}$-long peptide (LP) induces DKK1-specific human Tcell responses from myeloma patients. Myeloma patient's peripheral blood mononuclear cells were stimulated weekly with $\mathrm{DKK} 1_{3-76^{-} \mathrm{LP}}$ plus IL-2 and IL-7 in vitro, and the frequency of DKK1-specific $\mathrm{CD} 4^{+}$and $\mathrm{CD} 8^{+}$IFN- $\gamma$-secreting cells was detected by intracellular staining assay. (A) Representative results of $T$ cells

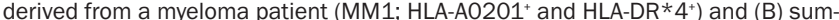
marized results from all ten myeloma patients at 4 weeks of in vitro stimulation. (C) Cytolytic activity of DKK1-specific T cells derived from MM1 against various target cells including autologous (MM1) or allogeneic (MM2 and MM3) plasma cells, myeloma cell lines, B cells, and K562 cells. MM1 and MM2 are HLA-A0201'; MM3 is $\mathrm{HLA}_{-\mathrm{A}} 1^{+}$and $\mathrm{A} 32^{+}$; U266 is HLA-A0201 ${ }^{+}$, ARP-1 is HLAA0201. Representative results from one of three experiments are shown. DC: dendritic cells.

synergy between the telomerase-specific Th responses with chemotherapy in lung cancer. ${ }^{26}$ The introduction of immunotherapy in clinical practice also emphasized the influence of immune responses on cancer prognosis and chemotherapy effectiveness. ${ }^{27,28}$ Politou et al. ${ }^{2}$ and Terpos et al. ${ }^{29,30}$ reported that serum concentration of DKK1 protein were increased in patients with MM and were correlated with severe bone disease. Autologous stem cell transplantation and chemotherapies with bortezomib, melphalan, dexamethasone and intermittent thalidomide significantly reduced serum DKK1 level and led to normalization of bone remodeling in relapsed myeloma. These pieces of evidence support the hypothesis that induction or augmentation of DKK1-specific Th1 cells by vaccination with DKK1 ${ }_{3-76}$-LP may improve the clinical outcome of cancer patients when combined with chemotherapy or other standard therapies. ${ }^{31,32} \mathrm{DKK}_{3-76}$-LP-specific Th responses in $\mathrm{MM}$ patients may positively influence overall survival. The impact of DKK1-specific Th responses on clinical outcome will be evaluated in future studies.

In conclusion, $\mathrm{DKK} 1_{3-76}$-LP provides a useful tool for

propagation of both DKK1-specific Th1 cells and CTL, and may synergize with CTL-epitopes to enhance cancer cell killing. These findings provide a rationale for a clinical trial of $\mathrm{DKK}_{3-76}$-LP -based immunotherapy against a broad spectrum of cancer types, as DKK1 is widely expressed by human cancer cells. ${ }^{19}$

\section{Disclosures}

No conflicts of interest to disclose.

\section{Contributions}

$J Q, R L$ and $Q Y$ initiated the study. JQ, RL and $C Z$ designed the experiments and wrote the paper; $R L$ and JQ performed most of the experiments and statistical analyses; $Q W$ prepared human samples; $E B$ helped with animal experiments; MY assisted in generating mice; JH and WF provided important suggestions.

\section{Funding}

This work was supported by Cleveland Clinic startup fund, VeloSano, the Leukemia and Lymphoma Society (6469-15), and the Multiple Myeloma Research Foundation.

\section{References}

1. Tian E, Zhan F, Walker $\mathrm{R}$, et al. The role of the Wnt-signaling antagonist DKK1 in the development of osteolytic lesions in multiple myeloma. N Engl J Med. 2003; 349(26):2483-2494.

2. Politou MC, Heath DJ, Rahemtulla A, et al. Serum concentrations of Dickkopf-1 protein are increased in patients with multiple myeloma and reduced after autologous stem cell transplantation. Int $\mathrm{J}$ Cancer. 2006;119(7):1728-1731
3. Kohn MJ, Kaneko KJ, DePamphilis MI DkkL1 (Soggy), a Dickkopf family member localizes to the acrosome during mammalian spermatogenesis. Mol Reprod Dev. 2005;71(4):516-522

4. Hall CL, Bafico A, Dai J, Aaronson SA, Keller ET. Prostate cancer cells promote osteoblastic bone metastases through Wnts. Cancer Res. 2005;65(17):7554-7560.

5. Qian J, Zheng Y, Zheng C, et al. Active vaccination with Dickkopf-1 induces protective and therapeutic antitumor immunity in murine multiple myeloma. Blood. 2012; 119(1):161-169.
6. Zwaveling S, Ferreira Mota SC, Nouta J, et al. Established human papillomavirus type 16-expressing tumors are effectively eradicated following vaccination with long peptides. J Immunol. 2002;169(1):350-358.

7. Vambutas A, DeVoti J, Nouri $M$, et al. Therapeutic vaccination with papillomavirus E6 and E7 long peptides results in the control of both established virus-induced lesions and latently infected sites in a preclinical cottontail rabbit papillomavirus model. Vaccine. 2005;23(45):5271-5280.

8. Disis ML, Wallace DR, Gooley TA, et al Concurrent trastuzumab and HER2/neu- 
specific vaccination in patients with metastatic breast cancer. J Clin Oncol. 2009; 27(28):4685-4692.

9. Brunsvig PF, Kyte JA, Kersten C, et al. Telomerase peptide vaccination in NSCLC: a phase II trial in stage III patients vaccinated after chemoradiotherapy and an 8-year update on a phase I/II trial. Clin Cancer Res. 2011;17(21):6847-6857.

10. Melief CJ, van der Burg SH. Immunotherapy of established (pre)malignant disease by synthetic long peptide vaccines. Nat Rev Cancer. 2008;8(5):351-360.

11. Qian J, Wang S, Yang J, et al. Targeting heat shock proteins for immunotherapy in multiple myeloma: generation of myeloma-specific CTLs using dendritic cells pulsed with tumor-derived gp96. Clin Cancer Res. 2005;11(24 Pt 1):8808-8815.

12. Yi Q, Bergenbrant S, Osterborg A, et al. Tcell stimulation induced by idiotypes on monoclonal immunoglobulins in patients with monoclonal gammopathies. Scand J Immunol. 1993;38(6):529-534.

13. Dabadghao S, Bergenbrant S, Anton D, He W, Holm G, Yi Q. Anti-idiotypic T-cell activation in multiple myeloma induced by $\mathrm{M}$ component fragments presented by dendritic cells. Br J Haematol. 1998;100(4):647-654.

14. Kim Y, Ponomarenko J, Zhu Z, et al. Immune epitope database analysis resource. Nucleic Acids Res. 2012;40(Web Server issue):W525-530.

15. Romani N, Reider D, Heuer $M$, et al. Generation of mature dendritic cells from human blood. An improved method with special regard to clinical applicability. J Immunol Methods. 1996;196(2):137-151.

16. Lutz MB, Kukutsch N, Ogilvie AL, et al. An advanced culture method for generating large quantities of highly pure dendritic cells from mouse bone marrow. J Immunol Methods. 1999;223(1):77-92.
17. Alexander J, Oseroff C, Dahlberg C, et al. A decaepitope polypeptide primes for multiple CD8+ IFN-gamma and Th lymphocyte responses: evaluation of multiepitope polypeptides as a mode for vaccine delivery. Immunol. 2002;168(12):6189-6198

18. Tangri S, Ishioka GY, Huang $X$, et al. Structural features of peptide analogs of human histocompatibility leukocyte antigen class I epitopes that are more potent and immunogenic than wild-type peptide. J Exp Med. 2001;194(6):833-846.

19. Qian J, Xie J, Hong S, et al. Dickkopf-1 (DKK1) is a widely expressed and potent tumor-associated antigen in multiple myeloma. Blood. 2007;110(5):1587-1594.

20. Yaccoby S, Ling W, Zhan F, Walker R, Barlogie B, Shaughnessy JD Jr. Antibodybased inhibition of DKK1 suppresses tumorinduced bone resorption and multiple myeloma growth in vivo. Blood. 2007 109(5):2106-2111.

25. Corbiere V, Chapiro J, Stroobant V, et al. Antigen spreading contributes to MAGE vaccination-induced regression of melanoma metastases. Cancer Res. 2011; 71(4):1253-1262.

22. Ribas A, Timmerman JM, Butterfield LH Economou JS. Determinant spreading and tumor responses after peptide-based cancer immunotherapy. Trends Immunol. 2003; 24(2):58-61

23. Castellino F, Germain RN. Cooperation between CD4+ and CD8+ T cells: when, where, and how. Annu Rev Immunol. 2006; 24:519-540.

24. Aarntzen EH, De Vries IJ, Lesterhuis WJ, et al. Targeting CD4(+) T-helper cells improves the induction of antitumor responses in dendritic cell-based vaccination. Cancer Res. 2013;73(1):19-29

25. Weide B, Zelba H, Derhovanessian E, et al. Functional $\mathrm{T}$ cells targeting NY-ESO-1 or
Melan-A are predictive for survival of patients with distant melanoma metastasis. J Clin Oncol. 2012;30(15):1835-1841.

26. Godet Y, Fabre E, Dosset M, et al. Analysis of spontaneous tumor-specific CD4 T-cell immunity in lung cancer using promiscuous HLA-DR telomerase-derived epitopes: potential synergistic effect with chemotherapy response. Clin Cancer Res. 2012; 18(10):2943-2953.

27. Kyte JA, Gaudernack G, Dueland S, Trachse S, Julsrud L, Aamdal S. Telomerase peptide vaccination combined with temozolomide: a clinical trial in stage IV melanoma patients. Clin Cancer Res. 2011;17(13):4568-4580.

28. Kantoff PW, Higano CS, Shore ND, et al Sipuleucel-T immunotherapy for castrationresistant prostate cancer. $\mathrm{N}$ Engl J Med. 2010;363(5):411-422.

29. Terpos E, Heath DJ, Rahemtulla A, et al Bortezomib reduces serum dickkopf-1 and receptor activator of nuclear factor-kappaB ligand concentrations and normalises indices of bone remodelling in patients with relapsed multiple myeloma. Br J Haematol. 2006;135(5):688-692.

30. Terpos E, Kastritis E, Roussou M, et al. The combination of bortezomib, melphalan, dexamethasone and intermittent thalidomide is an effective regimen for relapsed/refractory myeloma and is associated with improvement of abnormal bone metabolism and angiogenesis. Leukemia. 2008;22(12):2247-2256.

31. Ding ZC, Zhou G. Cytotoxic chemotherapy and CD4+ effector T cells: an emerging alliance for durable antitumor effects. Clin Dev Immunol. 2012;2012:890178.

32. Walter S, Weinschenk T, Stenzl A, et al. Multipeptide immune response to cancer vaccine IMA901 after single-dose cyclophosphamide associates with longer patient survival. Nat Med. 2012;18(8):1254-1261. 